\title{
TWO THEOREMS CONCERNING CONVERGENCE OF FOURIER SERIES
}

\section{SHIN-ICHI IZUMI ${ }^{1}$}

This paper contains two theorems concerning the convergence of Fourier series at a point. The theorems are independent of each other.

1. The Lebesgue convergence criterion reads as follows [5]:

Theorem 1. If we put $\phi_{x}(t)=f(x+t)+f(x-t)-2 s$ and suppose that

$$
\frac{1}{h} \int_{0}^{h} \phi_{x}(t) d t \rightarrow 0
$$

and

$$
\int_{h}^{\pi} \frac{\left|\phi_{x}(t)-\phi_{x}(t+h)\right|}{t} d t \rightarrow 0 \quad \text { as } h \rightarrow 0,
$$

then the Fourier series of $f(t)$ converges to $s$ at $t=x$.

The first condition (1) was generalized by many writers. We shall give here another generalization of (1), namely

Theorem 2. If

$$
\frac{1}{h} \int_{0}^{h}\left(\phi_{x}(t)-\phi_{x}(t+h)\right) d t \rightarrow 0 \quad \text { as } h \rightarrow 0
$$

and (2) holds, then the Fourier series of $f(t)$ converges at $t=x$.

Evidently (1) implies (3). Conditions (2) and (3) are independent of $s$ and then $\phi_{x}(t)$ may be replaced by $f(x+t)+f(x-t)$. That is, Theorem 2 is a "convergence criterion" but not a "criterion for convergence to $s$." Of course, Theorem 1 may be written as a criterion of the former type but there are many criteria of the latter type.

Received by the editors September 2, 1958 and, in revised form, November 21, 1958.

1 This research was supported in part by the United States Air Force under Contract AF 18(600)-1111 monitored by the Air Force Office of Scientific Research of the Air Research and Development Command, and in part by the National Science Foundation.

The author expresses his hearty thanks to Dr. M. Noble for his kindness in giving many suggestions. 
If we write

$$
\Phi(t)=\int_{0}^{t} \phi_{x}(u) d u
$$

and

$$
\begin{aligned}
& \Delta_{h} \Phi(x)=\Phi(x+h)-\Phi(x), \\
& \Delta_{h}^{2} \Phi(x)=\Phi(x+2 h)-2 \Phi(x+h)+\Phi(x),
\end{aligned}
$$

then (3) becomes

$$
h^{-1} \Delta_{h}^{2} \Phi(0) \rightarrow 0
$$

as $h \rightarrow 0$.

This means that $\Phi(x)$ is smooth at $x=0$. On the other hand condition (1) may be written as

$$
h^{-1} \Phi(h)=h^{-1} \Delta_{h} \Phi(0) \rightarrow 0
$$
as $h \rightarrow 0$.

This means that $\Phi(t)$ is differentiable to zero at $t=0$.

Theorem 2 follows from

TheOREM 3. If $\int_{-\pi}^{\pi} \phi_{x}(t) d t=0$, then conditions (2) and (3) imply (1).

Therefore Theorems 1 and 2 are equivalent, even though (3) (or (4)) is more general than (1) (or (5)).

We shall now prove Theorem 3 . Let $L_{n}(t)$ be an even function with period $2 \pi$ and let

$$
\begin{array}{ll}
L_{n}(t)=1 / 2 \pi & \text { on }(0,2 \pi / n), \\
L_{n}(t)=\pi / n t^{2} & \text { on }(2 \pi / n, \pi)(n>2) .
\end{array}
$$

Then $\int_{-\pi}^{\pi} L_{n}(t) d t=1$. We set $\phi_{x}(t)=\phi(t)$ and $h=\pi / n$ for the sake of simplicity and let

$$
\tau_{n}(t)=\int_{-\pi}^{\pi} L_{n}(u) \phi(t+u) d u
$$

We write

$$
\begin{gathered}
\phi(t)=\left[\phi(t)-\tau_{n}(t)\right]+\tau_{n}(t) \\
\int_{0}^{h} \phi(t) d t=\int_{0}^{h}\left[\phi(t)-\tau_{n}(t)\right] d t+\int_{0}^{h} \tau_{n}(t) d t=I+J .
\end{gathered}
$$

Let us show that $I=o(h)$ and $J=o(h)$ as $h \rightarrow 0$.

Now we write 


$$
\begin{aligned}
& I=\int_{0}^{h} d t \int_{-\pi}^{\pi} L_{n}(u)[\phi(t)-\phi(t+u)] d u \\
& =\int_{0}^{h} d t \int_{0}^{\pi} d u+\int_{0}^{h} d t \int_{-\pi}^{0} d u \\
& =\int_{0}^{h} d t \int_{0}^{\pi} L_{n}(u)[2 \phi(t)-\phi(t+u)-\phi(t-u)] d u \\
& =\int_{0}^{h} d t \sum_{k=0}^{n-1} \int_{k h}^{(k+1) h} L_{n}(u)[2 \phi(t)-\phi(t+u)-\phi(t-u)] d u \\
& =\int_{0}^{h} d t \sum_{k=0}^{n-1} \int_{0}^{h} L_{n}(u+k h)[2 \phi(t)-\phi(t+u+k h)-\phi(t-u-k h)] d u \\
& =\int_{0}^{h} d t \int_{0}^{h} L_{n}(u)[2 \phi(t)-\phi(t+u)-\phi(t-u)] d u \\
& +\sum_{k=1}^{n-1} \int_{0}^{h} L_{n}(u+k h) d u \int_{0}^{h}[2 \phi(t)-\phi(t+u+k h) \\
& -\phi(t-u-k h)] d u \\
& =I_{1}+I_{2} \text {, }
\end{aligned}
$$

say, where

$$
\begin{aligned}
I_{1} & =\frac{1}{2 \pi} \int_{0}^{h} d u \int_{0}^{h}[2 \phi(t)-\phi(t+u)-\phi(t-u)] d t \\
& =-\frac{1}{2 \pi} \int_{0}^{h}[\Phi(h+u)-2 \Phi(h)-\Phi(h-u)] d u \\
& =-\frac{1}{2 \pi} \int_{0}^{h} \Delta_{u}^{2} \Phi(h-u) d u=o(h)
\end{aligned}
$$

by continuity of $\Phi(x)$ and

$$
\begin{aligned}
I_{2}= & \sum_{k=1}^{n-1} \int_{0}^{h} L_{n}(u+k h) d u \int_{0}^{h}[\phi(t)-\phi(t+u+k h)] d t \\
& +\sum_{k=1}^{n-1} \int_{0}^{h} L_{n}(u+k h) d u \int_{0}^{h}[\phi(t)-\phi(t-u-k h)] d t \\
= & I_{21}+I_{22},
\end{aligned}
$$




$$
\begin{aligned}
I_{21}= & \sum_{k=1}^{n-1} \int_{0}^{h} L_{n}(u+k h) d u \int_{0}^{h}\{[\phi(t)-\phi(t+h)] \\
& +[\phi(t+h)-\phi(t+2 h)] \\
& +\cdots+[\phi(t+(k-1) h)-\phi(t+k h)] \\
& +[\phi(t+k h)-\phi(t+k h+u)]\} d t \\
= & \sum_{k=1}^{n-1} \int_{0}^{h} L_{n}(u+k h) d u\left\{\int_{0}^{k h}[\phi(t)-\phi(t+h)] d t\right. \\
& \left.\quad+\int_{k h}^{(k+1) h}[\phi(t)-\phi(t+u)] d t\right\} \\
= & I_{211}+I_{212},
\end{aligned}
$$$$
I_{22}=\sum_{k=1}^{n-1} \int_{0}^{h} L_{n}(u+k h) d u \int_{0}^{h}\{[\phi(t)-\phi(t-h)]
$$$$
+[\phi(t-h)-\phi(t-2 h)]
$$$$
+\cdots+[\phi(t-(k-1) h)-\phi(t-k h)]
$$$$
+[\phi(t-k h)-\phi(t-k h-u)]\} d t
$$$$
=\sum_{k=1}^{n-1} \int_{0}^{h} L_{n}(u+k h) d u\left\{\int_{0}^{(k-1) h}[\phi(t)-\phi(t+h)] d t\right.
$$$$
\left.+\int_{(k-1) h}^{k h}[\phi(t)-\phi(t+u)] d t\right\}
$$

$$
=I_{221}+I_{222}
$$

since

$$
\int_{0}^{h}[\phi(t)-\phi(t-h)] d t=0
$$

$$
\begin{aligned}
& \int_{0}^{h}[\phi(t-j h)-\phi(t-(j+1) h)] d t \\
&=\int_{0}^{h}[\phi(t+(j-1) h)-\phi(t+j h)] d t
\end{aligned}
$$

and

$$
\int_{0}^{h}[\phi(t-k h)-\phi(t-k h-u)] d t=\int_{(k-1) h}^{k h}[\phi(t)-\phi(t+u)] d t .
$$

We have 


$$
\begin{aligned}
I_{211} & =\sum_{k=1}^{n-1} \int_{0}^{h} L_{n}(u+k h) d u \sum_{j=0}^{k-1} \int_{j h}^{(j+1) h}[\phi(t)-\phi(t+h)] d t \\
& =\sum_{k=1}^{n-1} \int_{0}^{h} L_{n}(u+k h) d u \int_{0}^{h}[\phi(t)-\phi(t+h)] d t+\sum_{k=1}^{n-1} \sum_{j=1}^{k-1} \\
& =I_{211}^{\prime}+I_{211}^{\prime \prime}
\end{aligned}
$$

where

$$
I_{211}^{\prime}=\int_{h}^{\pi} L_{n}(u) d u \int_{0}^{h}[\phi(t)-\phi(t+h)] d t .
$$

Similarly putting $I_{221}=I_{221}^{\prime}+I_{221}^{\prime \prime}$, we have

$$
\begin{aligned}
I_{211}^{\prime}+I_{221}^{\prime} & =\left\{\frac{1}{2}\left(1-\frac{1}{n}\right)+\frac{1}{2}\left(1-\frac{2}{n}\right)\right\} \int_{0}^{h}[\phi(t)-\phi(t+h)] d t \\
& =\left(1-\frac{3}{2 n}\right) \Delta_{h}^{2} \Phi(0)=o(h)
\end{aligned}
$$

by (3). Further

$$
\begin{aligned}
I_{211}^{\prime \prime} & =\sum_{k=1}^{n-1} \sum_{j=1}^{k-1}=\sum_{j=1}^{n-2} \int_{j h}^{(j+1) h}[\phi(t)-\phi(t+h)] d t \sum_{k=0 j+1}^{n-1} \int_{0}^{h} L_{n}(u+k h) d u \\
& =\sum_{j=1}^{n-2} \int_{j h}^{(j+1) h}[\phi(t)-\phi(t+h)] d t \int_{(j+1) h}^{\pi} L_{n}(u) d u, \\
\left|I_{211}^{\prime \prime}\right| & \leqq \frac{\pi}{n} \sum_{j=1}^{n-2} \frac{1}{(j+1) h}\left|\int_{j h}^{(j+1) h}[\phi(t)-\phi(t+h)] d t\right| \\
& \leqq 2 h \int_{h}^{\pi} \frac{|\phi(t)-\phi(t+h)|}{t} d t=o(h)
\end{aligned}
$$

by (2).

A similar estimate holds for $I_{221}^{\prime \prime}$. We have

$$
I_{212}=\sum_{k=1}^{n-1} \int_{0}^{h} L_{n}(u+k h) d u \int_{k h}^{(k+1) h}[\phi(t)-\phi(t+u)] d t
$$

and then

$$
\begin{aligned}
\left|I_{212}\right| & \leqq \frac{\pi}{n} \sum_{k=1}^{n-1} \int_{0}^{h} \frac{k h}{(u+k h)^{2}} d u \int_{k h}^{(k+1) h} \frac{|\phi(t)-\phi(t+u)|}{t} d t \\
& \leqq A \int_{0}^{h} d u \sum_{k=1}^{n-1} \frac{1}{k} \int_{k h}^{(k+1) h} \frac{|\phi(t)-\phi(t+u)|}{t} d t
\end{aligned}
$$


which is $o(h)$ by Abel's lemma and condition (2). A similar estimate holds for $I_{222}$.

It remains to estimate $J$. Let us put $L_{n}^{*}(t)=L_{n}(t)-1 / 2 \pi$; then

$$
\int_{u}^{\pi} L_{n}^{*}(t) d t=0 \quad(0 \leqq u \leqq 2 h)
$$

since $L_{n}^{*}(t)=0$ for $0 \leqq t \leqq 2 h$. By $\int_{-\pi}^{\pi} \phi(u) d u=0$, we get

$$
\begin{aligned}
J & =\int_{0}^{h} \tau_{n}(t) d t=\int_{0}^{h} d t \int_{-\pi}^{\pi} \phi(u) L_{n}^{*}(u-t) d u \\
& =\int_{-\pi}^{\pi} \phi(u) d u \int_{0}^{h} L_{n}^{*}(u-t) d t \\
& =\int_{-\pi}^{\pi} \phi(u) d u \int_{u-h}^{u} L_{n}^{*}(t) d t=\int_{-\pi}^{\pi} \phi(u) d u\left(\int_{u-h}^{\pi}-\int_{u}^{\pi}\right) L_{n}^{*}(t) d t \\
& =\int_{-\pi}^{\pi} \phi(u+h) d u \int_{u}^{\pi} L_{n}^{*}(t) d t-\int_{-\pi}^{\pi} \phi(u) d u \int_{u}^{\pi} L_{n}^{*}(t) d t \\
|J| & \leqq \int_{-\pi}^{\pi}|\phi(u+h)-\phi(u)| d u\left|\int_{|u|}^{\pi} L_{n}^{*}(t) d t\right|
\end{aligned}
$$

and then

$$
|J| \leqq A h \int_{h}^{x} \frac{|\phi(u)-\phi(u+h)|}{u} d u=o(h) .
$$

Thus the theorem is proved.

There is an example ${ }^{2}$ of a function which satisfies condition (1) but not (2). Putting $\phi_{1}(u)=u^{-1} \Phi(u)$, it is sufficient to find a function $\phi_{1}(u)$ such that (i) $u \phi_{1}(u)$ is absolutely continuous, (ii) $\phi_{1}(2 u)-\phi_{1}(u)$ $\rightarrow 0$ as $u \rightarrow 0$, but (iii) $\phi_{1}(u)$ does not converge as $u \rightarrow 0$.

Let $\phi_{1}(u)$ be a continuous and differentiable function in the interval $(\pi / 2, \pi)$ such that the differential coefficient is bounded and $\phi_{1}(u)$ takes values 0 and 1 . We put

$$
\phi_{1}(u)=\phi_{1}\left(2^{k} u\right) \text { for }\left(\pi / 2^{k+1}, \pi / 2^{k}\right), \quad k=1,2,3, \cdots .
$$

Then $\phi_{1}(u)$ is defined in the interval $(0, \pi)$. Evidently

$$
\phi_{1}(2 u)-\phi_{1}(u)=0 \text { in }(0, \pi / 2)
$$

and then (ii) is satisfied, but $\phi_{1}(u)$ takes values 0 and 1 in the interval $\left(\pi / 2^{k+1}, \pi / 2^{k}\right), k=1,2,3, \cdots$, so that (iii) is also satisfied. Now

2 This example is due to Professor Kakutani, to whom the author expresses his hearty thanks. 


$$
\left(u \phi_{1}(u)\right)^{\prime}=\phi_{1}(u)+u \phi_{1}^{\prime}(u)
$$

where $\phi_{1}(u)$ is bounded and then integrable. For $u$ in the interval $\left(\pi / 2^{k+1}, \pi / 2^{k}\right)$,

$$
u \phi_{1}^{\prime}(u)=u\left(\phi_{1}\left(2^{k} u\right)\right)^{\prime}=2^{k} u \phi_{1}^{\prime}\left(2^{k} u\right)=v \phi_{1}^{\prime}(v)
$$

where $v=2^{k} u$ lies in the interval $(\pi / 2, \pi)$, and then $u \phi_{1}^{\prime}(u)$ is bounded in the interval $(0, \pi)$ and hence is integrable. Thus condition (i) is satisfied.

Finally we remark that Theorem 3 belongs to the same category as the Hille-Klein theorem [1] and its proof is similar to theirs (cf. [2]).

2. M. E. Noble [4] proved the following theorem which contains a well known convergence criterion of Hardy and Littlewood and a lacunary analogue suggested by R. P. Boas.

Theorem 1. Suppose that $f(t)$ is an integrable periodic function and

$$
\int_{0}^{t}|f(x+u)-f(x)| d u=o(t \phi(t))
$$

where $\phi(t)=O(1 / \log (1 / t))$ as $t \downarrow 0$ and is an increasing continuous function of $t$ when $t \geqq 0$ with $\phi(0)=0$. Suppose further that there are sequences of integers $\left(n_{k}\right)$ and $\left(\lambda_{n}\right)$ such that

$$
\int_{1 / n_{k}}^{1 / \lambda_{n k}} \frac{\phi(u)}{u} d u=O(1)
$$

and

$$
\liminf _{\boldsymbol{k} \rightarrow \infty} \min _{n_{k}-\lambda_{n_{k}}<n<m<n_{k}+\lambda_{n_{k}}}\left[s_{m}(x ; f)-s_{n}(x ; f)\right] \geqq 0 .
$$

Then $s_{n_{k}}(x ; f) \rightarrow f(x)$ as $k \rightarrow \infty$.

Noble raised the problem: whether condition (2) could be weakened to

$$
\limsup _{k \rightarrow \infty} \int_{1 / n_{k}}^{1 / \lambda_{n_{k}}} \frac{\phi(u)}{u} d u=+\infty
$$

and he proved that it cannot when there is a constant $\lambda>0$ such that

$$
\liminf _{t \downarrow 0}\{\phi(k t) / \phi(t)\} \geqq \lambda
$$

for any $k, 0<k<1$. 
Here we shall prove

THEOREM 2. Suppose that $\phi(t)$ is an increasing continuous function of $t$ when $t \geqq 0$ with $\phi(0)=0$ and that $\left(\lambda_{n}\right)$ is a sequence of positive numbers such that $\lambda_{n}=o(n)$ and

$$
\limsup _{n \rightarrow \infty} \int_{1 / n}^{1 / \lambda_{n}} \frac{\phi(t)}{t} d t=\infty .
$$

Then there are a continuous function $f(x)$ and a sequence $\left(n_{k}\right)$ such that

$$
\begin{gathered}
f(t)=o(\phi(t)) \text { as } t \rightarrow 0, \\
\lim _{k \rightarrow \infty} \max _{n_{n_{k}}<\lambda_{n_{k}}<<_{n}<m<\lambda_{n_{k}}+\lambda_{n_{k}}}\left|s_{m}(0 ; f)-s_{n}(0 ; f)\right|=0
\end{gathered}
$$

and

$$
s_{n_{k}}(0 ; f) \rightarrow \infty .
$$

Proof. ${ }^{3}$ We can suppose that $\lambda_{n} \rightarrow \infty$ as $n \rightarrow \infty$. Let $f(t)$ be an even function such that

$$
\begin{aligned}
f(t) & =c_{k} \phi(t) \sin n_{k} t & & \text { for } t \in I_{k}=\left(1 / n_{k}, 1 / \lambda_{n_{k}}\right) \\
& =0 & & \text { otherwise }
\end{aligned}
$$

where $\left(n_{k}\right)$ is taken such that the intervals $I_{k}$ do not overlap and

$$
\int_{I_{k}} \frac{\phi(t)}{t} d t \rightarrow \infty \quad \text { as } k \rightarrow \infty
$$

and $\left(c_{k}\right)$ is a sequence of positive numbers. The sequence $\left(n_{k}\right)$ and $\left(c_{k}\right)$ will be determined later. We have

$$
|f(t)| \leqq c_{k} \phi(t) \quad \text { for } t \in\left(1 / n_{k}, 1 / n_{k-1}\right)
$$

and then (5) holds if $c_{k} \rightarrow 0$. Now

$$
\begin{aligned}
s_{n_{k}}(0 ; f) & =\frac{2}{\pi} \int_{0}^{\pi} f(t) \frac{\sin n_{k} t}{t} d t+o(1) \\
& =\frac{2}{\pi} \sum_{j=1}^{\infty} c_{j} \int_{I} \phi(t) \sin n_{j} t \frac{\sin n_{k} t}{t} d t+o(1)=\frac{2}{\pi} \sum_{j=1}^{\infty} d_{j}+o(1) .
\end{aligned}
$$

We have

$$
d_{k}=c_{k}\left\{\frac{1}{2} \int_{I_{k}} \frac{\phi(t)}{t} d t-\frac{1}{2} \int_{I_{k}} \frac{\phi(t)}{t} \cos 2 n_{k} t d t\right\}
$$

where 
$\left|\int_{I_{k}} \frac{\phi(t)}{t} \cos 2 n_{k} t d t\right| \leqq n_{k} \phi\left(1 / \lambda_{n_{k}}\right)\left|\int_{I_{k}^{\prime}} \cos 2 n_{k} t d t\right|=o(1)\left(I_{k}^{\prime} \subset I_{k}\right)$.

Therefore we get

$$
d_{k} \geqq A c_{k} \int_{I_{k}} \frac{\phi(t)}{t} d t
$$

which tends to infinity by (8), if $c_{k}$ tends sufficiently slowly to zero.

Now, for $j<k$, if the sequence $\left(n_{k}\right)$ has the Hadamard gap condition, then

$$
\begin{aligned}
d_{j} & =c_{j} \int_{I_{j}} \frac{\phi(t)}{t} \sin n_{j} t \sin n_{k} t d t \\
& =\frac{1}{2} c_{j} \int_{I_{j}} \frac{\phi(t)}{t}\left(\cos \left(n_{k}-n_{j}\right) t-\cos \left(n_{k}+n_{j}\right) t\right) d t \\
\left|d_{j}\right| & \leqq \frac{1}{2} c_{j} n_{j} \phi\left(1 / \lambda_{n_{j}}\right)\left|\int_{I_{j}^{\prime \prime}}\left(\cos \left(n_{k}-n_{j}\right) t-\cos \left(n_{k}+n_{j}\right) t\right) d t\right| \\
& \leqq A c_{j} n_{j} / n_{k}
\end{aligned}
$$

where $I_{j}^{\prime \prime} \subset I_{j}$, and hence

$$
\sum_{j=1}^{k-1}\left|d_{j}\right| \leqq A \sum_{j=1}^{k-1} c_{j} n_{j} / n_{k}=o(1)
$$

if $\left(n_{k}\right)$ has Hadamard gaps.

For $j>k$, we have $\left|d_{j}\right| \leqq A \phi\left(1 / \lambda_{n_{j}}\right)$. If the sequence $\left(n_{k}\right)$ increases sufficiently rapidly so that $\sum \phi\left(1 / \lambda_{n_{j}}\right)<\infty$, then

$$
\sum_{j=k+1}^{\infty}\left|d_{j}\right|=o(1)
$$

Equation (7) follows from (9), (10) and (11).

It remains to prove (6). Let $n_{k}-\lambda_{n_{k}}<n<m<n_{k}+\lambda_{n_{k}}$. Then

$$
\begin{aligned}
& \frac{\pi}{2}\left(s_{m}(0 ; f)-s_{n}(0 ; f)\right)=\int_{0}^{\pi} f(t) \frac{\sin m t-\sin n t}{t} d t+o(1) \\
& =\sum_{j=1}^{\infty} c_{j} \int_{I_{j}} \phi(t) \sin n_{j} t \frac{\sin m t-\sin n t}{t} d t+o(1) \\
& =2 \sum_{j=1}^{\infty} c_{j} \int_{I_{j}} \frac{\phi(t)}{t} \sin n_{j} t \sin \frac{1}{2}(m-n) t \cos \frac{1}{2}(m+n) t d t+o(1) \\
& =2 \sum_{j=1}^{\infty} e_{j}+o(1) .
\end{aligned}
$$


We have

$$
\begin{aligned}
e_{j} & =c_{j} \int_{1 / n_{j}}^{1 / \lambda_{n_{j}}} \frac{\phi(t)}{t} \sin n_{j} t \sin \frac{1}{2}(m-n) t \cos \frac{1}{2}(m+n) t d t, \\
\left|e_{j}\right| & \leqq c_{j} \frac{m-n}{2} \int_{1 / n_{j}}^{1 / \lambda_{n_{j}}} \phi(t) d t \\
& \leqq c_{j} \frac{\lambda_{n_{k}}}{\lambda_{n_{j}}} \phi\left(1 / \lambda_{n_{j}}\right) .
\end{aligned}
$$

This gives, as for $d_{j}$,

$$
\sum_{j=k+1}^{\infty}\left|e_{j}\right|=o(1)
$$

when $\left(n_{k}\right)$ has sufficiently large gaps, since $\lambda_{i} \rightarrow \infty$ and $\phi(t) \rightarrow 0$ as $t \rightarrow 0$.

On the other hand,

$$
e_{j}=c_{j} \int_{I_{j}} \frac{\phi(t)}{t} \sin n_{j} t \sin m t d t-c_{j} \int_{I_{j}} \frac{\phi(t)}{t} \sin n_{j} t \sin n t d t .
$$

If $j \leqq k-1$, then $n_{j}$ is less than $m$ and $n$, so that, if $\left(n_{k}\right)$ has sufficiently large gaps, the estimate for $d_{j}$ to deduce (10) is now used to get

$$
\sum_{j=1}^{k-1}\left|e_{j}\right|=o(1)
$$

Finally it remains to prove that $e_{k}=o(1)$. We can suppose that $n=n_{k}$ and $m=n_{k}+\mu_{k}>n_{k}$. Then

$$
\begin{aligned}
e_{k} & =c_{k} \int_{I_{k}} \frac{\phi(t)}{t} \sin n_{k} t\left(\sin \left(n_{k}+\mu_{k}\right) t-\sin n_{k} t\right) d t \\
& =2 c_{k} \int_{I_{k}} \frac{\phi(t)}{t} \sin n_{k} t \cos \left(n_{k}+\mu_{k} / 2\right) t \sin \left(\mu_{k} / 2\right) t d t \\
& =2 c_{k} \sin \frac{\mu_{k}}{2 \lambda_{n_{k}}} \phi\left(1 / \lambda_{n_{k}}\right) \int_{1 / n_{k}^{\prime}}^{1 / \lambda_{n_{k}}} \frac{\sin \left(2 n_{k}+\mu_{k} / 2\right) t-\sin \mu_{k} t / 2}{t} d t
\end{aligned}
$$

where $n_{k}>n_{k}^{\prime}>\lambda_{n_{k}}$, since $\sin \mu_{k} t / 2$ is monotone in the interval $I_{k}$. Therefore $e_{k}=o(1)$. Thus the theorem is proved.

\section{REFERENCES}

1. E. Hille and G. Klein, On the Riemann's theorem of Fourier series, Duke Math. J. vol. 21 (1954). 
2. S. Izumi, Some trigonometrical series XIV, Proc. Japan Acad. vol. 31 (1955).

3. - Notes on Fourier analysis (XXVI): Negative examples in the theory of Fourier series, Tôhoku Math. J. vol. 1 (1950).

4. M. E. Noble, On a convergence criterion of Hardy and Littlewood, Quart. J. Math. vol. 9 (1958) pp. 28-39.

5. A. Zygmund, Trigonometrical series, Warsaw, 1936.

UNiversity OF ChiCAGo AND

NORTHWESTERN UNIVERSITY

\section{AN EXTREMAL PROBLEM FOR POLYNOMIALS}

FRANCIS P. CALLAHAN, JR.

Problem. Consider the class of $n$th order polynomials $\{f(z)\}$ such that $f(1)=0,|f(z)| \leqq 1$ for $|z|=1$. From this class select that polynomial for which

$$
\frac{1}{2 \pi} \int_{0}^{2 \pi}\left|f\left(e^{i \theta}\right)\right|^{2} d \theta \text { is greatest. }
$$

For the solution we require the following

Lemma. Let $h(z)=\sum_{-N}^{N} h_{n} z^{n},\left(\bar{h}_{n}=h_{-n}\right)$. Then there exists a polynomial $f(z)$ of degree $N$ such that $h(z)=|f(z)|^{2}$ for $|z|=1$ if and only if $h(z) \geqq 0$ for $|z|=1$. Proof is available in reference [1].

The function $1-|f(z)|^{2}$ (with $\bar{z}$ replaced by $1 / z$ ) satisfies the conditions of the lemma for any $f(z)$ that satisfies the conditions of the problem. Thus, we can write, $1-|f(z)|^{2}=|g(z)|^{2}$, where $g(z)$ satisfies the conditions that $|g(z)| \leqq 1$ for $|z|=1$ and $|g(1)|=1$. (Without real loss of generality, we take this last to mean $g(1)=1$.)

In addition, for $f(z)$ to solve the problem, the associated $g(z)$ must minimize the integral

$$
\frac{1}{2 \pi} \int_{0}^{2 \pi}\left|g\left(e^{i \theta}\right)\right|^{2} d \theta
$$

Writing $g(z)=\sum_{0}^{N} g_{n} z^{n}$, we see that we are seeking to minimize the quantity $\sum_{0}^{N}\left|g_{n}\right|^{2}$ subject to the constraint that $\sum_{0}^{N} g_{n}=1$. A straightforward application of the Schwarz Inequality vields: $1=\sum_{0}^{N} g_{n} \leqq\left(\sum_{0}^{N}\left|g_{n}\right|^{2}\right)^{1 / 2}(N+1)^{1 / 2}$. The sum-of-squares is smallest when we set $g_{n}=1 /(N+1)$, and obtain for the corresponding $g(z)$,

Received by the editors November 21, 1958 and, in revised form, January 15, 1959. 